\title{
Computing Approximation GCD of Several Polynomials by Structured Total Least Norm*
}

\author{
Xuefeng Duan ${ }^{1}$, Xinjun Zhang ${ }^{1}$, Qingwen Wang ${ }^{2}$ \\ ${ }^{1}$ College of Mathematics and Computational Science, Guilin University of Electronic Technology, Guilin, China \\ ${ }^{2}$ Department of Mathematics, Shanghai University, Shanghai, China \\ Email: zhangxinjunguilin@163.com
}

Received September 28, 2013; revised October 30, 2013; accepted November 8, 2013

Copyright (c) 2013 Xuefeng Duan et al. This is an open access article distributed under the Creative Commons Attribution License, which permits unrestricted use, distribution, and reproduction in any medium, provided the original work is properly cited.

\begin{abstract}
The task of determining the greatest common divisors (GCD) for several polynomials which arises in image compression, computer algebra and speech encoding can be formulated as a low rank approximation problem with Sylvester matrix. This paper demonstrates a method based on structured total least norm (STLN) algorithm for matrices with Sylvester structure. We demonstrate the algorithm to compute an approximate GCD. Both the theoretical analysis and the computational results show that the method is feasible.
\end{abstract}

Keywords: Sylvester Matrix; Approximate Greatest Common Divisor; Low Rank Approximation; Structured Total Least Norm; Numerical Method

\section{Introduction}

Let $\operatorname{deg}(f(x))$ be the degree of $f(x)$ and $C[x]$ be the set of univariate polynomials. $\|A\|_{2}$ stands for the spectral norm of the matrix $A . C^{n}$ and $C^{m \times n}$ are the vector spaces of complex $n$ vectors and $m \times n$ matrices, respectively. Transpose matrices and vectors are denoted by $A^{T}$ and $u^{T}$. GCD $(f, g)$ denotes the greatest common divisor for the polynomials $f$ and $g$. We use $\operatorname{rank}(A)$ to stand for the rank of matrix A.

$$
\operatorname{deg}\left(G C D\left(f_{1}(x)+\Delta f_{1}(x), f_{2}(x)+\Delta f_{2}(x), \cdots, f_{t}(x)+\Delta f_{t}(x)\right)\right) \geq k,
$$

and

$$
\left\|\Delta f_{1}(x)\right\|_{2}^{2}+\left\|\Delta f_{2}(x)\right\|_{2}^{2}+\cdots+\left\|\Delta f_{t}(x)\right\|_{2}^{2}
$$

is minimized.

The problem of computing approximate GCD of several polynomials is widely applied in speech encoding and filter design [1], computer algebra [2] and signal processing [3] and has been studied in [4-7] in recent years.

\footnotetext{
*The author is supported by a grant from National Natural Science Foundation of China (11101100; 11391240185; 11261014; 11226323) and Natural Science Foundation of Guangxi Province (No.2012GXN SFBA053006; 2013GX NSFBA019009; 2011GXNSFA018138).
}

In this paper, we consider the following problem. Let $f_{1}(x), \quad f_{2}(x), \cdots, \quad f_{t}(x) \in C[x] \backslash\{0\}$, namely

$$
\begin{gathered}
f_{1}(x)=a_{n} x^{n}+a_{n-1} x^{n-1}+\cdots+a_{1} x+a_{0}, \\
f_{i}(x)=b_{i p} x^{p}+b_{i(p-1)} x^{p-1}+\cdots+b_{i 1} x+b_{i 0}, i=2, \cdots, t .
\end{gathered}
$$

Problem 1.1. Set $k$ be a positive integer with $k \leq \min (n, p)$. We wish to compute $\Delta f_{1}(x), \Delta f_{2}(x)$, $\cdots, \Delta f_{t}(x) \in C[x] \backslash\{0\}$, such that

$$
\operatorname{deg}\left(\Delta f_{1}(x)\right) \leq n, \operatorname{deg}\left(\Delta f_{i}(x)\right) \leq p, 2 \leq i \leq t,
$$

Several methods to the problem have been presented. The generally-used computational method is based on the truncated singular decomposition(TSVD) [8] which may not be appropriate when a matrix has a special structure since they do not preserve the special structure (for example, Sylvester matrix). Another common method based on QR decomposition $[9,10]$ may suffer from loss of accuracy when it is applied to ill-conditioned problems and the algorithm derived in [11] can produce a more accurate result for ill-conditioned problems. Cadzow algorithm [12] is also a popular method to solve this problem which has been rediscovered in the literature [13]. 
Somehow it only finds a structured low rank matrix that is nearby a given target matrix but certainly is not the closet even in the local sense. Another method is based on alternating projection algorithm [14]. Although the algorithm can be applied to any low rank and any linear structure, the speed may be very slow. Some other methods have been proposed such as the ERES method [15], STLS method [16] and the matrix pencil method [17]. An approach to be described is called Structured Total Least Norm (STLN) which has been described for Hankel structure low rank approximation $[18,19]$ and Sylvester structure low rank approximation with two polynomials [20]. STLN is a problem formulation for obtaining an approximate solution

$(A+E) X=B+H$ to an overdetermined linear system $A X=B$ preserving the given structure in $A$ or $\left[\begin{array}{ll}A & B\end{array}\right]$.

In this paper, we apply the algorithm to compute the structured preserving rank reduction of Sylvester matrix. We introduce some notations and discuss the relationship between the GCD problems and low rank approximation of Sylvester matrices in Section 2. Based on STLN method, we describe the algorithm to solve Problem 1.1 in Section 3. In Section 4, we use some examples to illustrate the method is feasible.

\section{Main Results}

First of all, we shall prove that Problem 1.1 always has a solution.

Theorem 2.1. Suppose that $f_{1}, f_{2}, \cdots, f_{t}$, $\operatorname{deg}\left(f_{1}\right), \operatorname{deg}\left(f_{2}\right), \cdots, \operatorname{deg}\left(f_{t}\right)$ and $k$ are defined as those in Problem 1.1. There exist $\hat{f}_{1}, \hat{f}_{i} \in C[x]$ with $\operatorname{deg}\left(\hat{f}_{1}\right) \leq n, \operatorname{deg}\left(\hat{f}_{i}\right) \leq p$ and $\operatorname{deg}\left(\operatorname{GCD}\left(\hat{f}_{1}, \hat{f}_{2}, \cdots, \hat{f}_{t}\right)\right) \geq k$ such that for all $\bar{f}_{1}$, $\bar{f}_{i} \in C[x] \quad$ with $\quad \operatorname{deg}\left(\bar{f}_{1}\right) \leq n, \quad \operatorname{deg}\left(\bar{f}_{i}\right) \leq p \quad$ and $\operatorname{deg}\left(\operatorname{GCD}\left(\bar{f}_{1}, \bar{f}_{2}, \cdots, \bar{f}_{t}\right)\right) \geq k, \quad 2 \leq i \leq t$.

We have

$$
\left\|\hat{f}_{1}-f_{1}\right\|_{2}^{2}+\left\|\hat{f}_{2}-f_{2}\right\|_{2}^{2}+\cdots+\left\|\hat{f}_{t}-f_{t}\right\|_{2}^{2} \leq\left\|\bar{f}_{1}-f_{1}\right\|_{2}^{2}+\left\|\bar{f}_{2}-f_{2}\right\|_{2}^{2}+\cdots+\left\|\bar{f}_{t}-f_{t}\right\|_{2}^{2}
$$

Proof. Let $h \in C[x]$ be monic with $\operatorname{deg}(h)=k$ and set $u_{i} \in C[x]$ with $\operatorname{deg}\left(u_{i}\right) \leq \operatorname{deg}\left(f_{i}\right)-k$. For the real and imaginary parts of the coefficients of $h$ and of $u_{i}$, $(1 \leq i \leq t)$. We are considered with the continuous objective function

$$
\begin{aligned}
F\left(h, u_{1}, u_{2}, \cdots, u_{t}\right)= & \left\|u_{1} h-f_{1}\right\|_{2}^{2}+\left\|u_{2} h-f_{2}\right\|_{2}^{2} \\
& +\cdots+\left\|u_{t} h-f_{t}\right\|_{2}^{2} .
\end{aligned}
$$

We will prove that the function has a value on a closed and bounded set of its real argument vector which is smaller than elsewhere. Consider $\bar{f}_{1}=a_{n} x^{n}$ and $\bar{f}_{i}=b_{i p} x^{p}$ with a GCD of degree $\geq k$ for $2 \leq i \leq t$. Clearly, any $h$ and $u_{i}$ with

$$
F\left(h, u_{1}, u_{2}, \cdots, u_{t}\right)>\left\|\bar{f}_{1}-f_{1}\right\|_{2}^{2}+\left\|\bar{f}_{2}-f_{2}\right\|_{2}^{2}+\cdots+\left\|\bar{f}_{t}-f_{t}\right\|_{2}^{2}
$$

can be discarded. So from above,we know that the coefficients of $u_{1} h, u_{2} h, \cdots, u_{t} h$ can be bounded and so can the coefficients of $h, u_{1}, u_{2}, \cdots, u_{t}$ by any polynomials factor coefficient bound. Thus the function's domain $F\left(h, u_{1}, u_{2}, \cdots, u_{t}\right)$ is restricted to a sufficiently large ball. It remains to exclude $u_{1}=u_{2}=\cdots=u_{t}=0$ as the minimal solution. We have

$$
\begin{aligned}
F(h, 0,0, \cdots, 0) & =\left\|f_{1}\right\|_{2}^{2}+\left\|f_{2}\right\|_{2}^{2}+\cdots+\left\|f_{t}\right\|_{2}^{2} \\
& >\left\|\bar{f}_{1}-f_{1}\right\|_{2}^{2}+\left\|\bar{f}_{2}-f_{2}\right\|_{2}^{2}+\cdots+\left\|\bar{f}_{t}-f_{t}\right\|_{2}^{2}
\end{aligned}
$$

In conclusion, the theorem is true.

Now we begin to solve Problem 1.1, we first define a $p \times(n+p)$ matrix associated with $f_{1}(x)$ as follows

$$
S_{1}=\left[\begin{array}{ccccccccc}
a_{n} & a_{n-1} & a_{n-2} & \cdots & a_{1} & a_{0} & 0 & \cdots & 0 \\
0 & a_{n} & a_{n-1} & \cdots & \cdots & a_{1} & a_{0} & \ddots & \\
\vdots & & \ddots & \ddots & & & \ddots & \ddots & 0 \\
0 & \cdots & 0 & a_{n} & a_{n-1} & \cdots & \cdots & a_{1} & a_{0}
\end{array}\right],
$$

and an $n \times(n+p)$ matrix associated with $f_{i}(x)$, $i=2,3, \cdots, t$ as

$$
S_{i}=\left[\begin{array}{cccccccccc}
b_{i p} & b_{i}(p-1) & b_{i}(p-2) & \cdots & b_{i 1} & b_{i 0} & 0 & \cdots & \cdots & 0 \\
0 & b_{i p} & b_{i}(p-1) & \cdots & \cdots & b_{i 1} & b_{i 0} & 0 & \cdots & 0 \\
\vdots & \ddots & \ddots & & & & \ddots & \ddots & & \vdots \\
\vdots & \ddots & \ddots & \ddots & & & & \ddots & \ddots & 0 \\
0 & \cdots & 0 & b_{i p} & b_{i}(p-1) & \cdots & \cdots & \cdots & b_{i 1} & b_{i 0}
\end{array}\right],
$$




$$
S=\left[\begin{array}{c}
S_{1} \\
S_{2} \\
\vdots \\
S_{t}
\end{array}\right]^{\mathrm{T}} \in C^{(p+n) \times((t-1) n+p)} .
$$

Deleting the last $k-1$ rows of $S$ and the last $k-1$ columns of coefficients of $f_{1}, f_{2}, \cdots, f_{t}$ separately is $S$, We get the $k$-th Sylvester matrix $S_{k} \in C^{(n+p-k+1) \times((t-1) n+p-t k+t)}$

$$
S_{k}=\left[\begin{array}{cccccccccc}
a_{n} & 0 & & b_{2 p} & & & \cdots & b_{t p} & & \\
a_{n-1} & \ddots & & b_{2(p-1)} & \ddots & & \cdots & b_{t(p-1)} & \ddots & \\
\vdots & \ddots & a_{n} & \vdots & \ddots & b_{2 p} & \vdots & \vdots & \ddots & b_{t p} \\
a_{0} & & a_{n-1} & b_{10} & & b_{2(p-1)} & \cdots & b_{t 0} & & b_{t(p-1)} \\
& \ddots & \vdots & & \ddots & \vdots & \vdots & & \ddots & \vdots \\
& & a_{0} & & & b_{20} & \cdots & & & b_{t 0}
\end{array}\right] .
$$

It is well-known that $\operatorname{deg}\left(G C D\left(f_{1} \cdots f_{t}\right)\right) \geq k$ if and only if $S_{k}\left(f_{1} \cdots f_{t}\right)$ has rank deficiency at least 1 . We have

$$
\begin{aligned}
& \min _{\operatorname{deg}\left(G C D\left(\bar{f}_{1} \cdots \bar{f}_{t}\right)\right) \geq k}\left\|\bar{f}_{1}-f_{1}\right\|_{2}^{2}+\left\|\bar{f}_{2}-f_{2}\right\|_{2}^{2}+\cdots+\left\|\bar{f}_{t}-f_{t}\right\|_{2}^{2} \\
& \Leftrightarrow \min _{\operatorname{dimNullspace}\left(\bar{s}_{k}\right) \geq 1}\left\|\bar{f}_{1}-f_{1}\right\|_{2}^{2}+\left\|\bar{f}_{2}-f_{2}\right\|_{2}^{2}+\cdots+\left\|\bar{f}_{t}-f_{t}\right\|_{2}^{2} .
\end{aligned}
$$

where $\bar{S}_{k}$ is the $k$-th Sylvester matrix generated by $\bar{f}_{1}, \bar{f}_{2}, \cdots, \bar{f}_{t}$.

From above, we know that (2.1) can be transformed to the low rank approximation of a Sylvester matrix.

If we use STLN [16] to solve the following overdetermined system

$$
A_{k} X=b_{k} \text {, }
$$

for $S_{k}=\left[\begin{array}{ll}b_{k} & A_{k}\end{array}\right]$, where $b_{k}$ is the first column of $S_{k}$ and $A_{k}$ are the remainder columns of $S_{k}$, then we get a minimal perturbation $\left[\begin{array}{ll}h_{k} & E_{k}\end{array}\right]$ of Sylvester structure satisfying

$$
b_{k}+h_{k} \in A_{k}+E_{k} \text {. }
$$

So the solution with Sylvester structure is $\bar{S}_{k}=\left[\begin{array}{ll}h_{k}+b_{k} & E_{k}+A_{k}\end{array}\right]$ and dimNullspace $\left(\bar{S}_{k}\right)>1$.

We will give the following example and theorem to explain why we choose the first column to form the overdetermined system.

Example 2.1. Suppose three polynomials are given

$$
\begin{gathered}
f_{1}(x)=x^{2}-1, \\
f_{2}(x)=x^{2}+x-2, \\
f_{3}(x)=x^{2}+2 x-3 .
\end{gathered}
$$

The matrix $S$ is the Sylvester matrix generated by $f_{1}(x), \quad f_{2}(x)$ and $f_{3}(x)$

$$
S=\left[\begin{array}{cccccc}
1 & 0 & 1 & 0 & 1 & 0 \\
0 & 1 & 1 & 1 & 2 & 1 \\
-1 & 0 & -2 & 1 & -3 & 2 \\
0 & -1 & 0 & -2 & 0 & -3
\end{array}\right]
$$

The matrix $S$ is partitioned as $S=\left[\begin{array}{ll}\hat{b}_{1} & \hat{A}_{1}\end{array}\right]$ or $S=\left[\begin{array}{ll}\bar{A}_{1} & \bar{b}_{1}\end{array}\right]$, where $\hat{b}_{1}$ is the first column of $S$, whereas $b_{1}$ is the last column of $S$.

The overdetermined system

$$
\hat{A}_{1} X=\hat{b}_{1}
$$

has a solution $X=\left[\begin{array}{lllll}1 & 1 & 1 & -1 & -1\end{array}\right]^{\mathrm{T}}$, while the system

$$
\bar{A}_{1} X=\bar{b}_{1}
$$

has no solution.

Theorem 2.2. Suppose that $f_{1}, f_{2}, \cdots, f_{t}$, $\operatorname{deg}\left(f_{1}\right), \operatorname{deg}\left(f_{2}\right), \cdots, \operatorname{deg}\left(f_{t}\right)$ and $k$ are defined as those in Problem 1.1 and $S_{k}$ is the $k$-th Sylvester matrix of $f_{1}, f_{2}, \cdots, f_{t}$. Then the following statements are equivalent.

1) $\operatorname{dimNullspace}\left(S_{k}\right) \geq 1$;

2) $A_{k} X=b_{k}$ has a solution, where $b_{k}$ is the first column of $S_{k}$ and $A_{k}$ are the remainder columns of $S_{k}$.

Proof. $\Leftarrow$ Suppose $A_{k} X=b_{k}$ has a nonzero solution, then $b_{k} \in \operatorname{Range}\left(A_{k}\right)$. Since $b_{k}$ is the first column of $S_{k}$, obviously, the dimension of the rank deficiency of $S_{k}=\left[\begin{array}{ll}b_{k} & A_{k}\end{array}\right]$ is at least 1 .

$\Rightarrow$ Suppose the rank deficiency of $S_{k}=\left[\begin{array}{ll}b_{k} & A_{k}\end{array}\right]$ is at least 1 and $D(x)=G C D\left(f_{1}, f_{2}, \cdots, f_{t}\right)$,

$f_{1}^{*}=f_{1} / D(x), \quad f_{2}^{*}=f_{2} / D(x), \quad \cdots, \quad f_{t}^{*}=f_{t} / D(x)$. Multiplying the vector $V=\left[x^{n+p-k}, x^{n+p-k-1}, \cdots x, 1\right]$ to the matrix $S_{k}$, then we obtain

$$
V S_{k}=\left[x^{p-k} f_{1}, x^{p-k-1} f_{1}, \cdots, f_{1}, x^{n-k} f_{2}, \cdots, f_{2}, \cdots, x^{n-k} f_{t}, \cdots, f_{t}\right] .
$$


Next we will prove that $A_{k} X=b_{k}$ has a solution. If we multiply the vector $V$ to two sides of the equation $A_{k} X=b_{k}$, it turns out to be

$$
\begin{aligned}
& {\left[x^{p-k-1} f_{1}, \cdots, f_{1}, x^{n-k} f_{2}, \cdots, f_{2}, \cdots, x^{n-k} f_{t}, \cdots, f_{t}\right] X} \\
& =x^{p-k} f_{1} .
\end{aligned}
$$

The solution $X$ of equation (2.3) is equal to the coefficients of polynomials $u_{1}, u_{2}, \cdots, u_{t}$ such that

$$
x^{p-k} f_{1}=u_{1} f_{1}+u_{2} f_{2}+\cdots+u_{t} f_{t} .
$$

We can get $\operatorname{deg}(D(x)) \geq k$ and $\operatorname{deg}\left(f_{1}^{*}\right) \leq n-k$, $\operatorname{deg}\left(f_{i}^{*}\right) \leq p-k, \quad(2 \leq i \leq t)$ from

$\operatorname{dimNullspace}\left(S_{k}\right) \geq 1$. Dividing $x^{p-k}$ by $f_{2}^{*}+\cdots+f_{t}^{*}$, we obtain a quotient $q$ and a remainder $m$ satisfy

$$
x^{p-k}=q\left(f_{2}^{*}+\cdots+f_{t}^{*}\right)+m .
$$

where $\operatorname{deg}(q) \leq \operatorname{deg}(D(x))-k, \quad \operatorname{deg}(m) \leq p-k-1$. Now we can get that

$$
u_{1}=p, u_{2}=m f_{1}^{*}, \cdots, u_{t}=m f_{1}^{*} .
$$

are solutions of Equation (2.3), since

$$
\begin{aligned}
& \operatorname{deg}\left(u_{1}\right) \leq \operatorname{deg}(D(x))-k, \\
\operatorname{deg}\left(u_{i}\right)= & \operatorname{deg}(q)+\operatorname{deg}\left(f_{1}^{*}\right) \\
\leq & \operatorname{deg}(D(x))-k+\operatorname{deg}\left(f_{1}^{*}\right) \leq n-k,
\end{aligned}
$$

and

$$
u_{1} f_{1}+u_{2} f_{2}+\cdots+u_{t} f_{t}=f q\left(f_{2}^{*}+\cdots+f_{t}^{*}\right)+f p=f x^{p-k} .
$$

Next, we will illustrate for any given Sylvester matrix, as long as all the elements are allowed to be perturbed, we can always find $k$-Sylvester structure matrices $\left[\begin{array}{ll}h_{k} & E_{k}\end{array}\right]$ satisfy $b_{k}+h_{k} \in \operatorname{Range}\left(A_{k}+E_{k}\right)$, where $b_{k}$ is the first column of $S_{k}$ and $A_{k}$ are the remainder column of $S_{k}$.

Theorem 2.3. Given the positive integer $n, p, t$, there exists a Sylvester matrix $S \in C^{(n+p) \times(p+(t-1) n)}$ with rank deficiency $\mathrm{k}$.

Proof. We can always find polynomials $f_{1}, f_{2}, \cdots, f_{t} \in C[x]$ with $\operatorname{deg}\left(f_{1}\right)=n$, $\operatorname{deg}\left(f_{i}\right)=p, 2 \leq i \leq t$ and $\operatorname{deg}\left(\operatorname{GCD}\left(f_{1}, f_{2}, \cdots, f_{t}\right)\right)=k$. Hence $S$ is the Sylvester matrix of $f_{1}, f_{2}, \cdots, f_{t}$ and its rank deficiency is $\mathrm{k}$.

Corollary 2.1. Given the positive integer $n, p, t$, and $k$-th Sylvester matrix $S_{k}=\left[\begin{array}{ll}A_{k} & b_{k}\end{array}\right]$, where $A_{k} \in C^{(n+p-k+1) \times(p+(t-1) n-t k+t)}, \quad b_{k} \in C^{(n+p-k+1) \times 1}$, it is always possible to find a $k$-th Sylvester structure perturbation $\left[\begin{array}{ll}h_{k} & E_{k}\end{array}\right]$ such that $b_{k}+h_{k} \in \operatorname{Range}\left(A_{k}+E_{k}\right)$.

\section{STLN for Overdetermined System with Sylvester Structure}

In this section, we will use STLN method to solve the overdetermined system

$$
A_{k} X=b_{k} \text {, }
$$

According to theorem 2.3 and corollary 2.1, we can always find Sylvester structure $\left[\begin{array}{ll}h_{k} & E_{k}\end{array}\right]$ with $h_{k}+b_{k} \in \operatorname{Range}\left(A_{k}+E_{k}\right)$. Next we will use STLN method to find the minimum solution.

First, we define the Sylvester structure preserving perturbation $\left[\begin{array}{ll}h_{k} & E_{k}\end{array}\right]$ of $S_{k}$

$$
\left[\begin{array}{ll}
h_{k} & E_{k}
\end{array}\right]=\left[\begin{array}{cccccccccc}
z_{1} & & & z_{n+2} & & & \cdots & z_{n+(t-2) p+t} & & \\
z_{2} & \ddots & & z_{n+3} & \ddots & & \cdots & z_{n+(t-2) p+t+1} & \ddots & \\
\vdots & \ddots & z_{1} & \vdots & \ddots & z_{n+2} & \vdots & \vdots & \ddots & Z_{n+(t-2) p+t} \\
Z_{n+1} & & z_{2} & z_{n+p+2} & & z_{n+3} & \cdots & Z_{n+(t-1) p+t} & & Z_{n+(t-2) p+t+1} \\
& \ddots & \vdots & & \ddots & \vdots & \vdots & & \ddots & \vdots \\
& & z_{n+1} & & & z_{n+p+2} & \cdots & & & Z_{n+(t-1) p+t}
\end{array}\right]
$$

can be represented by a vector

$$
Z=\left[z_{1}, z_{2}, \cdots, z_{(n+(t-1) p+t)}\right]^{\mathrm{T}} .
$$

We can define a matrix $P_{k}$ such that $h_{k}=P_{k} Z$.

$$
P_{k}=\left[\begin{array}{cc}
I_{n+1} & 0 \\
0 & 0
\end{array}\right] \in C^{(n+p-k+1) \times(n+(t-1) p+t)},
$$

where $I_{n+1}$ is a $(n+1) \times(n+1)$ identity matrix.

We will solve the equality-constrained least squares problem

$$
\min _{Z, X}\|Z\|_{2}^{2} \text {, subject to } r=0 \text {. }
$$

where the structured residual $r$ is

$$
r=r(Z, X)=b_{k}+h_{k}-\left(A_{k}+E_{k}\right) X .
$$

By using the penalty method, the formulation (3.1) can be transformed into

$$
\min _{Z, X}\left\|\begin{array}{c}
w r(Z, X) \\
Z
\end{array}\right\|_{2}^{2}, w \gg 1,
$$


where $w$ is a large penalty value.

Let $\Delta Z$ and $\Delta X$ stand for a small change in $Z$ and $X$, respectively and $\Delta E_{k}$ be the corresponding change in $E_{k}$. Then the first order approximate to $r(Z+\Delta Z, X+\Delta X)$ is

$$
\begin{aligned}
r(Z+\Delta Z, X+\Delta X)= & b_{k}+P_{k}(Z+\Delta Z) \\
& -\left(A_{k}+E_{k}+\Delta E_{k}\right)(X+\Delta X) \\
\approx & b_{k}+P_{k} Z-\left(A_{k}+E_{k}\right) X+P_{k} \Delta Z \\
& -\left(A_{k}+E_{k}\right) \Delta X-\Delta E_{k} X .
\end{aligned}
$$

Introducing a matrix of Sylvester structure $Y_{k}$ and

$$
X=\left[x_{1}, x_{2}, \cdots, x_{p+(t-1) n-t k+t-1}\right]^{\mathrm{T}}
$$

(3.2) can be approximated by

$$
\min _{\Delta X, \Delta Z}\left\|\left[\begin{array}{cc}
w\left(Y_{k}-P_{k}\right) & w\left(A_{k}+E_{k}\right) \\
I_{n+(t-1) p+t} & 0
\end{array}\right]\left[\begin{array}{c}
\Delta Z \\
\Delta X
\end{array}\right]+\left[\begin{array}{c}
-w r \\
Z
\end{array}\right]\right\|_{2}
$$

where $Y_{k} \in C^{(n+p-k+1) \times(n+(t-1) p+t)}$ satisfies that

$$
Y_{k} Z=E_{k} X .
$$

In the following, we present a method to obtain the matrix $Y_{k}$. Suppose $f_{1}, f_{2}, \cdots, f_{t}, E, \quad Z$ and $X$ are defined as above. Multiplying the vector

$$
V=\left[x^{n+p-k}, x^{n+p-k-1}, \cdots, x, 1\right],
$$

to the two sides of equation (3.4), it becomes

$$
V Y_{k} Z=V E_{k} X \text {. }
$$

$$
\begin{aligned}
\text { Let } \hat{X} & =\left[\begin{array}{l}
0 \\
X
\end{array}\right] \text {, we obtain } \\
V E_{k} X & =V\left[\begin{array}{ll}
h_{k} & E_{k}
\end{array}\right] \hat{X}=\hat{g}_{1} \hat{u}_{1}+\hat{g}_{2} \hat{u}_{2}+\cdots+\hat{g}_{t} \hat{u}_{t},
\end{aligned}
$$

where $\hat{g}_{1}$ is the polynomial with degree $n$ which is generated by the subvector of $Z$ :

$$
\left[\begin{array}{llll}
z_{1} & z_{2} & \cdots & z_{n+1}
\end{array}\right]
$$

$\hat{g}_{2}$ is the polynomial with degree $p$ which is generated by the subvector of $Z$ :

$$
\left[\begin{array}{llll}
Z_{n+2} & Z_{n+3} & \cdots & Z_{n+p+2}
\end{array}\right],
$$

$\hat{g}_{t}$ is the polynomial with degree $p$ which is generated by the subvector of $Z$ :

$$
\left[\begin{array}{llll}
Z_{n+(t-2) p+t} & Z_{n+(t-2) p+t+1} & \cdots & Z_{n+(t-1) p+t}
\end{array}\right],
$$

$\hat{u}_{1}$ is the polynomial with degree $p-k-1$ which is generated by the subvector of $X$ :

$$
\left[\begin{array}{llll}
0 & x_{1} & \cdots & x_{p-k}
\end{array}\right]
$$

$\hat{u}_{2}$ is the polynomial with degree $n-k$ which is generated by the subvector of $X$ :

$$
\left[\begin{array}{llll}
x_{p-k+1} & x_{p-k+2} & \cdots & x_{n+p-2 k+1}
\end{array}\right]
$$

$\hat{u}_{t}$ is the polynomial with degree $n-k$ which is generated by the subvector of $X$ :

$$
\left[\begin{array}{llll}
x_{p+(t-2) n-(t-1) k+t-1} & x_{p+(t-2) n-(t-1) k+t} & \cdots & x_{p+(t-1) n-t k+t-1}
\end{array}\right],
$$

Here we will present a simple example to illustrate how to find $Y_{k}$.

Example 3.1. Suppose $k=1, \quad X=\left[x_{1}, x_{2}, \cdots, x_{7}\right]$ and $f_{1}(x)=a_{3} x^{3}+a_{2} x^{2}+a_{1} x+a_{0}$,

$$
\begin{aligned}
& f_{2}(x)=b_{22} x^{2}+b_{21} x+b_{20}, \\
& f_{3}(x)=b_{32} x^{2}+b_{31} x+b_{30} .
\end{aligned}
$$

$$
A=\left[\begin{array}{ccccccc}
0 & b_{22} & 0 & 0 & b_{32} & 0 & 0 \\
a_{3} & b_{21} & b_{22} & 0 & b_{31} & b_{32} & 0 \\
a_{2} & b_{20} & b_{21} & b_{22} & b_{30} & b_{31} & b_{32} \\
a_{1} & 0 & b_{20} & b_{21} & 0 & b_{30} & b_{31} \\
a_{0} & 0 & 0 & b_{20} & 0 & 0 & b_{30}
\end{array}\right], b_{1}=\left[\begin{array}{c}
a_{3} \\
a_{2} \\
a_{1} \\
a_{0} \\
0
\end{array}\right],
$$

then

$$
Y_{1}=\left[\begin{array}{cccccccccc}
0 & 0 & 0 & 0 & x_{2} & 0 & 0 & x_{5} & 0 & 0 \\
x_{1} & 0 & 0 & 0 & x_{3} & x_{2} & 0 & x_{6} & x_{5} & 0 \\
0 & x_{1} & 0 & 0 & x_{4} & x_{3} & x_{2} & x_{7} & x_{6} & x_{5} \\
0 & 0 & x_{1} & 0 & 0 & x_{4} & x_{3} & 0 & x_{7} & x_{6} \\
0 & 0 & 0 & x_{1} & 0 & 0 & x_{4} & 0 & 0 & x_{7}
\end{array}\right] .
$$

\section{Approximate GCD Algorithm and Experiments}

The following algorithm is designed to solve Problem 1.1 .

\section{Algorithm 4.1.}

Input-A Sylvester matrix $\mathrm{S}$ generated by $f_{1}, f_{2}$, $\cdots, f_{t}$, respectively, an integer $k$ and a tolerance tol .

Output-Polynomials $\bar{f}_{1}, \bar{f}_{2}, \cdots, \bar{f}_{t}$ and the Euclidean distance $\left\|\bar{f}_{1}-f_{1}\right\|_{2}^{2}+\left\|\bar{f}_{2}-f_{2}\right\|_{2}^{2}+\cdots+\left\|\bar{f}_{t}-f_{t}\right\|_{2}^{2}$ is to a minimum.

1) Form the $k$-th Sylvester matrix $S_{k}$ as the above section, set the first column of $S_{k}$ as $b_{k}$ and the remainder columns of $S_{k}$ as $A_{k}$. Let $E_{k}=0, h_{k}=0$.

2) Calculate $X$ from $\min \left\|A_{k} X-b_{k}\right\|_{2}$ and $r=b_{k}-A_{k} X$. Compute $P_{k}$ and $Y_{k}$ as the above section.

3) Repeat

$$
\text { (1) } \min _{\Delta X, \Delta Z}\left\|\left[\begin{array}{cc}
w\left(Y_{k}-P_{k}\right) & w\left(A_{k}+E_{k}\right) \\
I_{n+(t-1) p+t} & 0
\end{array}\right]\left[\begin{array}{l}
\Delta Z \\
\Delta X
\end{array}\right]+\left[\begin{array}{c}
-w r \\
Z
\end{array}\right]\right\|_{2} \text {. }
$$


(2) Let $X=X+\Delta X, Z=Z+\Delta Z$,

(3) Form the matrix $E_{k}$ and $h_{k}$ from $Z$, and $Y_{k}$ from $X$. Let $A_{k}=A_{k}+E_{k}$,

$b_{k}=b_{k}+h_{k}, \quad r=b_{k}-A_{k} X$ until $\|\Delta X\|_{2} \leq$ tol and $\|\Delta Z\|_{2} \leq$ tol

4) Output the polynomials $\bar{f}_{1}, \bar{f}_{2}, \ldots, \bar{f}_{t}$ constructed from $\left[\begin{array}{ll}b_{k} & A_{k}\end{array}\right]$.

Given a tolerance $\varepsilon$, we can use the Algorithm 4.1 to compute an approximate GCD of $f_{1}, f_{2}, \cdots, f_{t}$. The method begin with $k \leq \min \{n, p\}$ using Algorithm 4.1 to compute the minimum perturbation $N=\sum_{i}\left\|\bar{f}_{i}-f_{i}\right\|_{2}^{2}$ with $\operatorname{dimNullspace}(S) \geq 1$. If $N<\varepsilon$, then we can compute the approximate GCD form matrix $S_{k}$. Otherwise, we reduce $k$ by one and repeat the Algorithm 4.1.

Example 4.1. We wish to find the minimal polynomial perturbations $\Delta f$ and $\Delta g$ of

$$
\begin{gathered}
f=x^{2}-6 x+5, \\
g=x^{2}-6.3 x+5.72,
\end{gathered}
$$

satisfy that the polynomials $\Delta f+f$ and $\Delta g+g$ have a common root. We take two cases into account.

Case 1: The leading coefficients can be perturbed. Let $k=1$ and $t o l=10^{-3}$, after 3 iterations, we get the polynomials $\bar{f}$ and $\bar{g}$

$$
\begin{aligned}
& \bar{f}=0.9850 x^{2}-6.0029 x+4.9994, \\
& \bar{g}=1.0150 x^{2}-6.2971 x+5.7206,
\end{aligned}
$$

with a minimum distance

$$
N=\|\bar{f}-f\|_{2}^{2}+\|\bar{g}-g\|_{2}^{2}=0.0004663 .
$$

Case 2: The leading coefficients can be perturbed. Let $k=1$ and tol $=10^{-3}$, after 3 iterations, we have the polynomials $\bar{f}$ and $\bar{g}$ :

$$
\begin{gathered}
\bar{f}=x^{2}-6.0750 x+4.9853, \\
\bar{g}=x^{2}-6.222 x+5.7353,
\end{gathered}
$$

with a minimum distance

$$
N=\|\bar{f}-f\|_{2}^{2}+\|\bar{g}-g\|_{2}^{2}=0.01213604583 .
$$

Example 4.2. Let $k=1$, tol $=10^{-3}$ and

$$
\begin{gathered}
f_{1}=1.98 x^{3}+5 x^{2}+5 x+2.96 \\
f_{2}=1.99 x^{2}-1.01+3.01 \\
f_{3}=2 x^{2}+4.99 x+2.99
\end{gathered}
$$

after 8 iterations, we have the polynomials

$$
\begin{gathered}
\bar{f}_{1}=1.9800 x^{3}+5.0000 x^{2}+5.0000 x+2.9600, \\
\bar{f}_{2}=1.9963 x^{2}+1.0100 x-3.0099,
\end{gathered}
$$

$$
\bar{f}_{3}=2.0071 x^{2}+4.9900 x+2.9902,
$$

with a minimum distance

$$
N=\left\|\bar{f}_{1}-f_{1}\right\|_{2}^{2}+\left\|\bar{f}_{2}-f_{2}\right\|_{2}^{2}+\left\|\bar{f}_{3}-f_{3}\right\|_{2}^{2}=9.0763 \times 10^{-5},
$$

and the CPU time

$$
t=0.974920(s)
$$

Example 4.3. Let $k=1$, $\quad$ tol $=10^{-3}$ and

$$
\begin{gathered}
f_{1}=1.85 x^{4}-2 x^{3}-2.69 x^{2}-1.42 x, \\
f_{2}=1.47 x^{3}-2.94 x^{2}+1.18 x-2.36, \\
f_{3}=0.52 x^{3}-4.01 x^{2}+5.94 x, \\
f_{4}=0.52 x^{3}-0.13 x^{2}-1.05 x-2.58 .
\end{gathered}
$$

after 11 iterations, we have the polynomials

$$
\begin{gathered}
\bar{f}_{1}=1.85 x^{4}-2 x^{3}-2.69 x^{2}-1.42 x, \\
\bar{f}_{2}=1.47 x^{3}-2.94 x^{2}+1.18 x-2.36, \\
\bar{f}_{3}=0.5242 x^{3}-4.01 x^{2}+5.9405 x, \\
\bar{f}_{4}=0.52 x^{3}-0.134 x^{2}+-1.05 x-2.58 .
\end{gathered}
$$

with a minimum distance

$$
\begin{aligned}
N & =\left\|\bar{f}_{1}-f_{1}\right\|_{2}^{2}+\left\|\bar{f}_{2}-f_{2}\right\|_{2}^{2}+\left\|\bar{f}_{3}-f_{3}\right\|_{2}^{2}+\left\|\bar{f}_{4}-f_{4}\right\|_{2}^{2} \\
& =3.4387 \times 10^{-5},
\end{aligned}
$$

and the CPU time

$$
t=0.642582(s) .
$$

Example 4.4. Let $k=2, \quad t o l=10^{-3}$ and

$$
\begin{gathered}
f_{1}=0.144 x^{4}-0.761 x^{3}+1.316 x^{2}-0.74 x, \\
f_{2}=0.393 x^{3}-2.212 x^{2}+4.132 x-2.56 \\
f_{3}=0.182 x^{3}-0.358 x^{2}-0.752 x+1.48, \\
f_{4}=0.173 x^{3}-0.544 x^{2}+0.01 x+0.592 .
\end{gathered}
$$

after 1 iteration, we have the polynomials

$$
\begin{gathered}
f_{1}=0.144 x^{4}-0.761 x^{3}+1.316 x^{2}-0.74 x, \\
f_{2}=0.393 x^{3}-2.212 x^{2}+4.132 x-2.56, \\
f_{3}=0.182 x^{3}-0.3548 x^{2}-0.752 x+1.48, \\
f_{4}=0.173 x^{3}-0.4701 x^{2}+0.01 x+0.6257 .
\end{gathered}
$$

with a minimum distance

$$
\begin{aligned}
N & =\left\|\bar{f}_{1}-f_{1}\right\|_{2}^{2}+\left\|\bar{f}_{2}-f_{2}\right\|_{2}^{2}+\left\|\bar{f}_{3}-f_{3}\right\|_{2}^{2}+\left\|\bar{f}_{4}-f_{4}\right\|_{2}^{2} \\
& =0.0066,
\end{aligned}
$$

and the CPU time 


$$
t=0.001335(s) .
$$

Example 4.5. Let $k=2, \quad t o l=10^{-3}$ and

$$
\begin{gathered}
f_{1}=0.64 x^{6}-2.56 x^{5}+2.56 x^{4} \\
+0.62 x^{3}-1.03 x^{2}-3.32 x+5.8 \\
f_{2}=1.85 x^{5}-5.7 x^{4}+1.31 x^{3}+3.96 x^{2}+2.84 \\
f_{3}=0.44 x^{5}-3.23 x^{4}+7.64 x^{3}-4.7 x^{2}-4.72 x+4.72, \\
f_{4}=1.23 x^{5}-4.92 x^{4}+2.65 x^{3}+9.645 x^{2}-11.12 x+2.04, \\
f_{5}=1.31 x^{5}-5.76 x^{4}+8.49 x^{3}-5.47 x^{2}-0.48 x+5.16
\end{gathered}
$$

after 1 iteration, we have the polynomials

$$
\begin{gathered}
f_{1}=0.6401 x^{6}-2.56 x^{5}+2.5603 x^{4} \\
+0.62 x^{3}-1.03 x^{2}-3.32 x+5.8, \\
f_{2}=1.85099 x^{5}-5.7 x^{4}+1.3102 x^{3}+3.96 x^{2}+2.84, \\
f_{3}=0.4389 x^{5}-3.23 x^{4}+7.64 x^{3} \\
-4.7003 x^{2}-4.72 x+4.72, \\
f_{4}=1.23 x^{5}-4.92 x^{4}+2.65 x^{3}+9.645 x^{2}-11.12 x+2.04, \\
f_{5}=1.307 x^{5}-5.76 x^{4}+8.4902 x^{3} \\
-5.47 x^{2}-0.48 x+5.16
\end{gathered}
$$

with a minimum distance

$$
\begin{aligned}
N= & \left\|\bar{f}_{1}-f_{1}\right\|_{2}^{2}+\left\|\bar{f}_{2}-f_{2}\right\|_{2}^{2}+\left\|\bar{f}_{3}-f_{3}\right\|_{2}^{2} \\
& +\left\|\bar{f}_{4}-f_{4}\right\|_{2}^{2}+\left\|\bar{f}_{5}-f_{5}\right\|_{2}^{2} \\
= & 1.1460 \times 10^{-5},
\end{aligned}
$$

and the CPU time

$$
t=0.0923583(s) \text {. }
$$

Examples 4.1, 4.2, 4.3, 4.4 and 4.5 show that Algorithm 4.1 is feasible to solve Problem 1.1.
In Table 1, we present the performance of Algorithm 4.1 and compare the accuracy of the new fast algorithm with the algorithms in $[9,21]$. Denote $n$ be the total degree of polynomials $f_{1}$ and $p$ be the total degree of polynomials $f_{i}, 2 \leq i \leq t$. It (Chu) stands for the number of iterations by the method in [14] whereas it (STLN) denotes the number of iterations by Algorithm 4.1. Denoted by error(Zeng) and error (STLN) are the perturbations $\sum_{i}\left\|\bar{f}_{i}-f_{i}\right\|_{2}^{2}$ computed by the method in [21] and Algorithm 4.1, respectively. The last two columns denote the CPU time in seconds costed by AFMP algorithm and our algorithm, respectively.

As shown in the above table, we show that our method based on STLN algorithm converges quickly to the minimal approximate solutions, needing no more than 2 iterations whereas the method in [14] requires more iteration steps. We also note that our algorithm still converges very quickly when the degrees of polynomials become large while the algorithm in [14] needs more iteration steps. Besides, our algorithm needs less CPU time than the AFMP algorithm. So the convergence speed of our method is faster. From the errors, we demonstrate that our method has smaller magnitudes compared with the method in [21]. So our algorithm can generate much more accurate solutions.

\section{Conclusion}

In this paper, we present that approximation GCD of several polynomials can be solved by a practical and reliable way based on STLN method and transformed to the approximation of Sylvester structure problem. For the matrices related to the minimization problems are all structured matrix with low displacement rank, applying the algorithm to solve these minimization problems would be possible. The complexity of the algorithm is reduced with respect to the degrees of the given polynomials. Although the problem of structured low rank ap-

Table 1. Algorithm performance on benchmarks.

\begin{tabular}{ccccccccc}
\hline Ex & $\mathrm{n}, \mathrm{p}$ & $\mathrm{k}$ & it (Chu) & it (STLN) & error (Zeng) & error (STLN) & time (s) (AFMP) & time (s) (STLN) \\
\hline 1 & 2,2 & 1 & 5 & 2 & $1.89 \mathrm{e}-4$ & $2.87 \mathrm{e}-5$ & 7.76 & 2.5 \\
2 & 3,3 & 2 & 8 & 2 & $1.36 \mathrm{e}-3$ & $1.05 \mathrm{e}-4$ & 19.51 & 7.81 \\
3 & 5,4 & 3 & 11 & 2 & $1 \mathrm{e}-3$ & $1.56 \mathrm{e}-6$ & 6.81 & 2.44 \\
4 & 6,6 & 4 & 23 & 2 & $1.46 \mathrm{e}-3$ & $1.96 \mathrm{e}-10$ & 31.829 & 10.08 \\
5 & 8,7 & 5 & 33 & 2 & $6.53 \mathrm{e}-4$ & $1.98 \mathrm{e}-16$ & 50.222 & 16.95 \\
6 & 10,10 & 6 & 43 & 2 & $1.61 \mathrm{e}-3$ & $1.51 \mathrm{e}-12$ & 157.09 & 61.40 \\
7 & 14,13 & 7 & 58 & 2 & $1.23 \mathrm{e}-3$ & $2.61 \mathrm{e}-4$ & 273.7 & 122.31 \\
8 & 28,28 & 10 & 634 & 2 & $2.6 \mathrm{e}-3$ & $3.54 \mathrm{e}-4$ & 559.3 & 210.65 \\
\hline
\end{tabular}


proximation has been studied in many literatures and obtained many accomplishments, there is still much work to be done, for example, low rank approximation of finite dimensional matrix has not been fully resolved.

\section{REFERENCES}

[1] B. DeMoor, “Total Least Squares for Affinely Structured Matrices and the Noisy Realization Problem," IEEE Transactions on Signal Process, Vol. 42, No. 11, 1994, pp. 3104-3113. http://dx.doi.org/10.1109/78.330370

[2] R. M. Corless, P. M. Gianni, B. M. Tragerm and S. M. Watt, "The Singular Value Decomposition for Polynomial System," Proceedings of International Symposium on Symbolic and Algebraic Computation, Montreal, 1995, pp. 195-207

[3] S. R. Khare, H. K. Pillai and M. N. Belur, "Numerical Algorithm for Structured Low Rank Approximation Problem," Proceeding of the 19th International Symposium on Mathematical Theory of Networks and Systems, Budapest, Hungary, 2010.

[4] E. Kaltofen, Z. F. Yang and L. H. Zhi, “Approximate Greatest Common Divisors of Several Polynomials with Linearly Constrained Coecients and Simgular Polynomials,” Proceedings of International Symposium on Symbolic and Algebraic Computations, Genova, 2006.

[5] N. Karkanias, S. Fatouros, M. Mitrouli and G. H. Halikias, "Approximate Greatest Common Divisor of Many Polynomials, Generalised Resultants, and Strength of Approximation," Computers \& Mathematics with Applications, Vol. 51, No. 12, 2006, pp. 1817-1830. http://dx.doi.org/10.1016/j.camwa.2006.01.010

[6] I. Markovsky, "Structured Low-Rank Approximation and Its Applications,” Automatica, Vol. 44, No. 4, 2007, pp. 891-909. http://dx.doi.org/10.1016/j.automatica.2007.09.011

[7] D. Rupprecht, "An Algorithm for Computing Certied Approximate GCD of Univariate Polynomials,” Journal of Pure and Applied Algebra, Vol. 139, No. 1-3, 1999, pp. 255-284. http://dx.doi.org/10.1016/S0022-4049(99)00014-6

[8] J. A. Cadzow, "Signal Enhancement: A Composite Property Mapping Algorithm,” IEEE Transactions on Acoustic Speech Signal Process, Vol. 36, No. 1, 1988, pp. 4962. http://dx.doi.org/10.1109/29.1488

[9] G. Cybenko, “A General Orthogonalization Technique with Applications to Time Series Analysis and Signal Processing," Mathematics of Computation, Vol. 40, 1983, pp. 323-336. http://dx.doi.org/10.1090/S0025-5718-1983-0679449-6

[10] J. R. Winkler and J. D. Allan, "Structured Total Least Norm and Approximate GCDs of Inexact Polynomials,”
Journal of Computational and Applied Mathematics, Vol. 215, No. 1, 2008, pp. 1-13.

http://dx.doi.org/10.1016/j.cam.2007.03.018

[11] A. Frieze, R. Kannaa and S. Vempala, "Fast Monte-Carlo Algorithm for Finding Low Rank Approximations," Journal of ACM, Vol. 51, No. 6, 2004, pp. 1025-1041. http://dx.doi.org/10.1145/1039488.1039494

[12] R. Beer, "Quantitative in Vivo NMR (Nuclear Magnetic Resonance on Living Objects),” University of Technology Delft, 1995.

[13] B. Paola, "Structured Matrix-Based Methods for Approximate Polynomial GCD,” Edizioni della Normale, 2011.

[14] M. T. Chu, R. E. Funderlic and R. J. Plemmons, "Structured Low Rank Approximation,” Linear Algebra Applications, Vol. 366, No. 1, 2003, pp. 157-172. http://dx.doi.org/10.1016/S0024-3795(02)00505-0

[15] B. Beckermann and G. Labahn, “A Fast and Numerically Stable Euclidean-Like Algorithm for Detecting Relative Prime Numerical Polynomials," IEEE Journal of Symbolic Computation, Vol. 26, No. 6, 1998, pp. 691-714. http://dx.doi.org/10.1006/jsco.1998.0235

[16] B. Y. Li, Z. F. Yang and L. H. Zhi, "Fast Low Rank Approximation of a Sylvester Matrix by Structured Total Least Norm,” Journal of JSSAC (Japan Society for Symbolic and Algebraic Computation), Vol. 11, No. 34, 2005, pp. 165-174.

[17] B. Botting, M. Giesbrecht and J. May, "Using Riemannian SVD for Problems in Approximate Algebra,” Proceedings of the 2005 International Workshop on Symbolic-Numeric, 2005, Xi'an.

[18] E. Kaltofen, Z. F. Yang and L. H. Zhi, "Structured Low Rank Approximation of a Sylvester Matrix,” International Workshop on Symbolic-Numeric Computation, Xi'an, 2005, pp. 19-21.

[19] H. Park, L. Zhang and J. B. Rosen, "Low Rank Approximation of a Hankel Matrix by Structured Total Least Norm,” BIT Numerical Mathematics, Vol. 39, No. 4, 1999, pp. 757-779. http://dx.doi.org/10.1023/A:1022347425533

[20] L. H. Zhi and Z. F. Yang, "Computing Approximate GCD of Univariate Polynomials by Structure Total Least Norm," Mathematics-Mechanization Research Preprints, No. 24, 2004, pp. 375-387.

[21] Z. Zeng and B. H. Dayton, "The Approximate GCD of Inexact Polynomials Part 2: A Multivariate Algorithm," Proceedings of the 2004 International Symposium on Symbolic and Algebraic Computation, Santander, 2004. 